\title{
Backprojection to image slip
}

\author{
Ryo Okuwaki, ${ }^{1}$ Amato Kasahara, ${ }^{2, \star}$ Yuji Yagi, ${ }^{3}$ Shiro Hirano, ${ }^{4}$ and Yukitoshi Fukahata ${ }^{5}$ \\ ${ }^{1}$ Graduate School of Life and Environmental Sciences, University of Tsukuba, Tsukuba, Ibaraki 305-8572, Japan. \\ E-mail: rokuwaki@gmail.com \\ ${ }^{2}$ Independent Researcher. \\ ${ }^{3}$ Faculty of Life and Environmental Sciences, University of Tsukuba, Tsukuba, Ibaraki 305-8572, Japan. \\ ${ }^{4}$ College of Science and Engineering, Ritsumeikan University, Kusatsu, Shiga 525-8577, Japan. \\ ${ }^{5}$ Disaster Prevention Research Institute, Kyoto University, Uji, Kyoto 611-0011, Japan.
}

This is a pre-copyedited, author-produced PDF of an article accepted for publication in Geophysical Journal International following peer review. The version of record; R Okuwaki, A Kasahara, Y Yagi, S Hirano, Y Fukahata; Backprojection to image slip, Geophysical Journal International, doi:10.1093/gji/ggx502, is available online at: https://doi .org/10.1093/gji/ggy505.

\section{SUMMARY}

Waveform backprojection is a key technique of earthquake-source imaging, which has been widely used for extracting information of earthquake source evolution that cannot be obtained by kinematic source inversion. The technique enjoys considerable popularity, owing to the simplicity of its implementation and the robustness of its processing, but the physical meaning of backprojection images has remained elusive. In this study, we reviewed the mathematical representation of backprojection (BP) and hybrid backprojection (HBP) methods, following the pioneering work of Fukahata et al. (Geophys. J. Int. (2014) 196, 552-559), to clarify the physical implications of BP images. We found that signal intensity in BP and HBP images is scaled with the amplitude of the Green's function that corresponds to a unit-step slip, which results in the signal intensity being depth dependent. We propose variants of BP and HBP, which we call kinematic BP and HBP, 
respectively, to relate the BP signal intensity to slip motion of an earthquake by modifying the normalizing factors used in the original BP and HBP methods. The original BP and HBP images remain useful for assessing the spatiotemporal strength of the wave radiation, which scales with the amplitude of the Green's function, whereas the kinematic BP and HBP methods are suitable for imaging the slip motion that is responsible for the high-frequency radiation produced during the source-rupture process.

Key words: Image processing; Time series analysis; Earthquake dynamics; Earthquake source observations; Theoretical seismology

\section{INTRODUCTION}

Waveform backprojection (BP) is a technique for tracking the source evolution of specific seismic phases (e.g. the $P$ phase) during an earthquake (see Kiser \& Ishii 2017, for a comprehensive review of BP). Owing to its robustness and applicability to high-frequency waveforms, BP has been used for extracting information about earthquake-rupture evolution that is missed by the analyses of low-frequency waveforms by kinematic source inversion, including, for example, multiple branching ruptures (Meng et al. 2012b; Satriano et al. 2012; Fan et al. 2016, 2017), triggering ruptures of very early aftershocks (D’Amico et al. 2010; Kiser \& Ishii 2013; Fan \& Shearer 2016), cascading asperity ruptures (Okuwaki et al. 2014, 2016), mechanisms of deep earthquakes (Suzuki \& Yagi 2011; Kiser et al. 2011; Ye et al. 2013), frequency-dependent wave radiation of subduction zone megathrust earthquakes (Kiser \& Ishii 2011; Wang \& Mori 2011; Yao et al. 2013; Satriano et al. 2014; Yin et al. 2016, 2018), and complex rupture evolution associated with the geometric features of a fault system (Meng et al. 2012a; Uchide et al. 2013; Vallée \& Satriano 2014; Okuwaki \& Yagi 2018).

According to theoretical studies and dynamic numerical simulations of rupture evolution, highfrequency waves are generated by abrupt changes of slip velocity or rupture velocity, or both (Madariaga 1977; Bernard \& Madariaga 1984; Spudich \& Frazer 1984; Madariaga et al. 2006; Beresnev 2017). Such changes can be caused by barriers or asperities, including fault roughness (Das \& Aki 1977; Aki 1979; Dunham et al. 2011; Shi \& Day 2013; Bruhat et al. 2016; Mai et al. 2017). By applying the BP technique, studies have imaged intense high-frequency radiation just before the rupture penetrates a large-slip area or geometric barriers (e.g. Uchide et al. 2013; Yagi \& Okuwaki 2015; Okuwaki \&

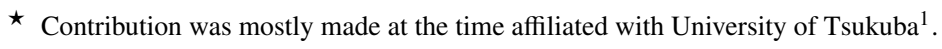


Yagi 2018), a finding that is consistent with our theoretical understanding of the generation of highfrequency radiation. In the case of some subduction-zone megathrust earthquakes, however, a distinct anti-correlation with respect to depth has been reported, between a shallow large-slip area and the deep sources of intense high-frequency radiation, which may reflect the rupture of heterogeneous, small high-stress patches; this finding leads to the idea that rupture properties within these subduction zones are segmented along depth (Koper et al. 2012; Lay et al. 2012; Ye et al. 2016). This idea may be useful for a unified understanding of subduction-zone megathrust earthquakes involving the apparent diversity of source rupture evolution.

However, the tendency of lacking intense high-frequency signal at the shallow part of the seismogenic zone generally observed through application of BP methods may not directly draw conclusion that the intensity of kinematic features responsible for high-frequency radiation increases with depth. Since the observations show that the rupture front often goes along both up-dip and down-dip directions (e.g. $M_{\mathrm{W}} 9.02011$ Tohoku-oki, Japan and $M_{\mathrm{W}} 8.32015$ Illapel, Chile, earthquakes; Meng et al. 2011; Melgar et al. 2016; Meng et al. 2018), it is plausible that there exist kinematic features around the rupture tip or the healing front (e.g. Madariaga et al. 2006) even at shallow. In fact, as shown in Section 2, BP and HBP images are not simple representation of kinematic features of the source process, and they have an inherent depth-dependent bias that is proportional to the amplitude of the Green's function.

In this study we propose an alternative BP technique that enables us to extract kinematic features of rupture evolution from high-frequency waveform data. We first review the mathematical representation of the BP method, following the pioneering work of Fukahata et al. (2014), who have clarified the theoretical background of the BP techniques and showed that a BP image can represent slip motion as either slip velocity or slip acceleration. However, because Fukahata et al. (2014) basically considered the case of a point source, they did not discuss in detail the normalizing factor adopted for the BP method. We point out the importance of the normalizing factor, which plays a critical role in the resultant signal intensity for the case of multiple-point sources. We consider both the original timedomain BP method (e.g. Ishii et al. 2005) and the Hybrid backprojection (HBP) method (Yagi et al. 2012; Okuwaki et al. 2014). The latter is a variant of the BP technique that introduces the Green's function to enhance the depth resolution of the projected images by using depth phases. We clarify the theoretical meaning of the signal intensity obtained by the BP and HBP methods, which depends on the amplitude of the Green's function and is not directly related to the slip motion. We examine this theoretical outcome through numerical tests using synthetic waveforms, and confirm that the images obtained by the BP and HBP methods have an inherent depth dependence, which is due to the normalizing factor. We propose new variants of the BP and HBP methods (which we call kinematic BP 
and HBP methods) that, by taking the effect of the normalizing factor into account, allow us to remove the depth-dependent bias in the original BP and HBP images and retrieve signal intensities that are related to slip velocity or slip acceleration. We test the methods through numerical experiments and application to the observed data of the $M_{\mathrm{W}} 8.32015$ Illapel, Chile, earthquake, and confirm that the relative signal intensity is related to the slip motion by the kinematic BP and HBP methods.

\section{DEPTH DEPENDENCE IN BP AND HBP IMAGES}

In this section, we investigate the mathematical expressions of the BP and HBP methods (Ishii et al. 2005, 2007; Yagi et al. 2012; Fukahata et al. 2014). Particularly, we consider the role of the normalizing factors in the implementations and their effect on the resultant signal intensity, which was not addressed in detail by Fukahata et al. (2014). We then perform a numerical test by using synthetic waveforms to demonstrate that both the BP and HBP images have an inherent depth dependence that is proportional to the amplitude of the Green's function for a unit-step slip.

\subsection{Representation of BP}

A BP image is made by stacking observed waveforms as follows:

$$
s_{i}^{\mathrm{BP}}(t)=\sum_{j} w_{j} \frac{u_{j}\left(t+t_{i j}^{p}\right)}{A_{j}},
$$

where $s_{i}^{\mathrm{BP}}(t)$ is the signal intensity of the BP image at the $i$ th source location at hypocentral time $t$ (where $t=0$ corresponds to the origin time of the earthquake), and $u_{j}\left(t+t_{i j}^{p}\right)$ is the observed waveform at the $j$ th station that is time-shifted by the theoretical travel time of the $P$ phase $t_{i j}^{p}$ between the $i$ th source location and the $j$ th station. For simplicity, we neglect the calibration of travel time error (Ishii et al. 2007; Fan \& Shearer 2017) and the slowness correction (Meng et al. 2016). $w_{j}$ is a weighting factor for each waveform, which is introduced to avoid spatial bias due to the station distribution; $w_{j}$ is usually designed to be negatively correlated with station density and sums to 1 , that is, $\sum_{j} w_{j}=1$ (details are shown in Section 2.3). The normalizing term $A_{j}$ is given by the root-sum-square of the observed waveform,

$$
A_{j}=\operatorname{pol}\left(u_{j}\right) \cdot \sqrt{\int_{0}^{T} u_{j}^{2}\left(\tau+t_{j}^{p}\right) d \tau},
$$

where $\operatorname{pol}\left(u_{j}\right) \in\{-1,+1\}$ is the polarity of the first motion of waveform $u_{j}, t_{j}^{p}$ is the $P$-phase arrival time at the $j$ th station, and $T$ is the waveform length. The normalizing factor can also be represented by the maximum amplitude of the observed waveform, although these forms of the normalizing factor were not presented explicitly in the relevant papers. In this study, we use the representation in eq. (2), 
because of its similarity to the normalizing factor in the HBP method defined in eq. (8). The observed waveform $u_{j}$ can be expressed as

$$
\begin{aligned}
u_{j}(t) & =\sum_{i^{\prime}}\left(a_{i^{\prime}} * G_{i^{\prime} j}\right)(t) \\
& =\left(a_{i} * G_{i j}\right)(t)+\sum_{i^{\prime}(\neq i)}\left(a_{i^{\prime}} * G_{i^{\prime} j}\right)(t),
\end{aligned}
$$

where $a_{i}$ is the slip motion at the $i$ th source location on the fault surface, $*$ denotes convolution, and we neglect the background noise of the observed waveform for simplicity. The Green's function $G_{i j}$ in eq. (3) is the deformation at the $j$ th station due to a unit-step slip on the $i$ th subfault. For the velocity data $u_{j}$, slip motion $a_{i}$ may represent slip velocity if the Green's function is a propagator from slip velocity to far-field velocity, or slip acceleration if the Green's function is a propagator from slip velocity to far-field displacement (Fukahata et al. 2014). Hereafter, we refer to the slip motion $a_{i}$ as slip velocity, considering together that the waveform $u_{j}$ is velocity data and the Green's function $G_{i j}$ is for unitstep slip velocity. By substituting eq. (3) into eq. (1), we get the following expression for the signal intensity,

$$
s_{i}^{\mathrm{BP}}(t)=\sum_{j} \frac{w_{j}}{A_{j}}\left(\left(a_{i} * G_{i j}\right)\left(t+t_{i j}^{p}\right)+\sum_{\left.i^{\prime} \neq i\right)}\left(a_{i^{\prime}} * G_{i^{\prime} j}\right)\left(t+t_{i j}^{p}\right)\right)
$$

As shown in eq. (4), eq. (1) is divided into two terms: the first term is the signal that corresponds to the contribution from the $i$ th source, and the second term is noise, which is the contribution from other $i^{\prime}$ th locations. If the noise term can be assumed to be suppressed by stacking (Fukahata et al. 2014) as,

$$
\sum_{j} \frac{w_{j}}{A_{j}}\left(\sum_{i^{\prime}(\neq i)}\left(a_{i^{\prime}} * G_{i^{\prime} j}\right)\left(t+t_{i j}^{p}\right)\right) \approx 0,
$$

then the signal intensity at the $i$ th location can ideally be represented as

$$
s_{i}^{\mathrm{BP}}(t) \approx \sum_{j} \frac{w_{j}}{A_{j}}\left(a_{i} * G_{i j}\right)\left(t+t_{i j}^{p}\right)
$$

where the signal intensity at the $i$ th source location $s_{i}^{\mathrm{BP}}$ represents the slip velocity at the $i$ th source location $a_{i}$, which scales with the normalizing factor $A_{j}$ and the Green's function $G_{i j}$. Hence, the signal intensity $s_{i}^{\mathrm{BP}}$ is not uniquely determined by the amplitude of the slip velocity at the $i$ th source location $a_{i}$, but is additionally controlled by a scaling factor composed of $A_{j}$ and $G_{i j}$, which depends on the source location because its numerator includes $G_{i j}$. 


\subsection{Representation of HBP}

The HBP image is made by stacking cross-correlation functions of the observed waveform and the Green's function:

$$
s_{i}^{\mathrm{HBP}}(t)=\sum_{j} w_{j} \frac{\left(u_{j} \hat{\times} G_{i j}^{p}\right)(t)}{A_{i j}}=\sum_{j} w_{j} \frac{\int_{0}^{T} u_{j}(\tau) G_{i j}^{p}(\tau-t) d \tau}{A_{i j}}
$$

where $\hat{x}$ denotes cross-correlation, and $G_{i j}^{p}$ is the theoretical Green's function that corresponds to the deformation due to a unit-step slip velocity. $T$ is the time period of the cross-correlation function of the waveform and the Green's function. The weighting factor $w_{j}$ is the same as in eq. (1), and the normalizing factor $A_{i j}$ is represented as

$$
A_{i j}=\sqrt{\int_{0}^{T} u_{j}^{2}\left(\tau+t_{j}^{p}\right) d \tau} \cdot \sqrt{\int_{0}^{T} G_{i j}^{p 2}\left(\tau+t_{i j}^{p}\right) d \tau},
$$

which is designed to normalize the cross-correlation function in the numerator of eq. (7). Eq. (3) can be used to expand eq. (7) into signal and noise terms as follows:

$$
s_{i}^{\mathrm{HBP}}(t)=\sum_{j} \frac{w_{j}}{A_{i j}}\left[\left(\left(a_{i} * G_{i j}\right) \hat{\times} G_{i j}^{p}\right)(t)+\sum_{i^{\prime}(\neq i)}\left(\left(a_{i^{\prime}} * G_{i^{\prime} j}\right) \hat{\times} G_{i j}^{p}\right)(t)\right] .
$$

If the noise term can be assumed to be suppressed by stacking (Fukahata et al. 2014) as

$$
\sum_{j} \frac{w_{j}}{A_{i j}}\left(\sum_{i^{\prime}(\neq i)}\left(a_{i^{\prime}} * G_{i^{\prime} j}\right) \hat{\times} G_{i j}^{p}\right)(t) \approx 0,
$$

the final form of the signal intensity is

$$
s_{i}^{\mathrm{HBP}}(t) \approx \sum_{j} \frac{w_{j}}{A_{i j}}\left(\left(a_{i} * G_{i j}\right) \hat{\times} G_{i j}^{p}\right)(t) .
$$

The resultant signal intensity at the $i$ th source location $s_{i}^{\mathrm{HBP}}$ represents the slip velocity $a_{i}$ scaled with a weighting factor $w_{j}$, the normalizing factor $A_{i j}$ and the cross-correlation function of the Green's functions. Eq. (11) indicates that the resultant signal intensity of the HBP method is also expected to correlate with the source location because the scaling factor includes the Green's functions.

\subsection{Numerical test}

As seen in eqs. (6) and (11), the signal intensity of the BP and HBP images is not a simple representation of the slip motion at the specific source location; but rather, it includes additional contributions from the normalizing factors and the Green's functions. We performed a numerical test with synthetic waveforms computed using a known slip velocity $a$ to empirically show how the scaling factors in eqs. (6) and (11) affect the resultant BP and HBP images. First, we generated synthetic waveforms using 20 point sources that were randomly distributed on a dipping model plane in the Chilean subduction zone 

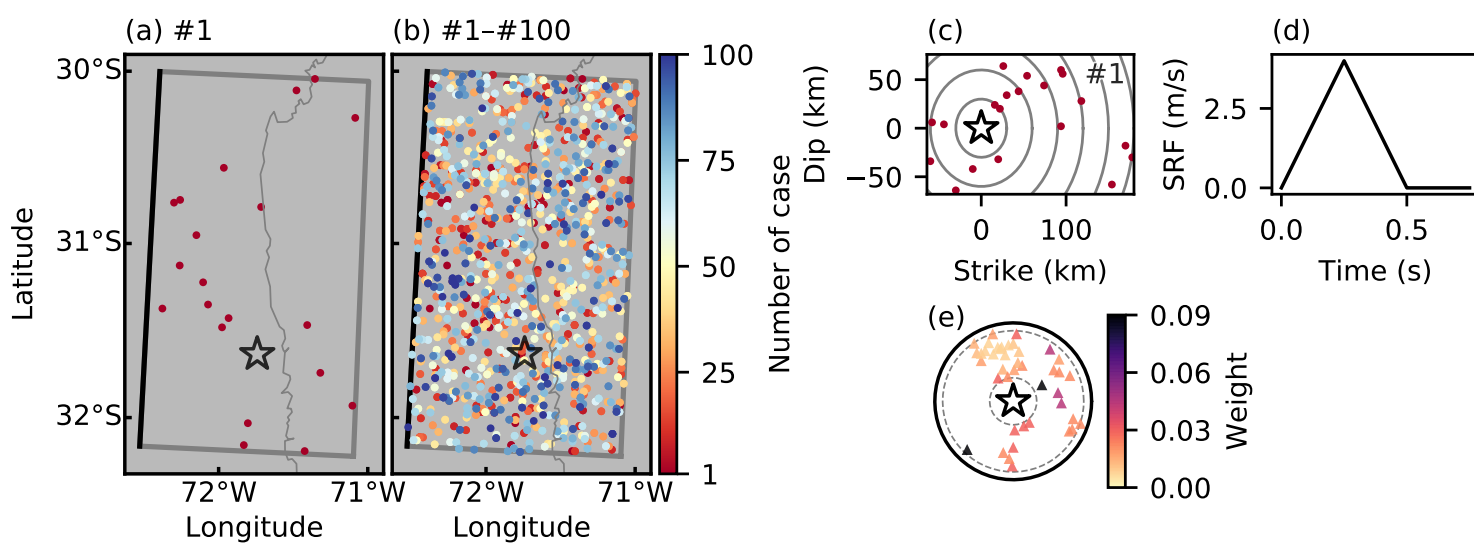

Figure 1. Model setting for the numerical test. (a) Map view of the distribution of randomly selected point sources for the case \#1 and (b) all the point sources through the cases \#1-\#100. The color of point sources represents the index of the case. The gray rectangle outlines the model fault plane with a dip angle of $15^{\circ}$, the black line on the west side is the up-dip limit of the fault area, and the star denotes the nucleation point (hypocentre). The thin gray curved line shows the coastline. (c) Relation between the point-source location (red dot) and the rupture front for the case 1. The star denotes the nucleation point. Gray circles represent the constantly propagating rupture front snapshotted at $10 \mathrm{~s}$ interval. (d) Slip-rate function (SRF) built in each point source. (e) Observation station distribution denoted as triangles. The star denotes the nucleation point, and the gray dashed circles show epicentral distances at $30^{\circ}$ and $90^{\circ}$. Color of each triangle represents the weight defined in eq. (12).

(Fig. 1a). Each point source has a uniform potency of $4 \times 10^{6} \mathrm{~m}^{3}\left(2 \times 2 \mathrm{~km}^{2}\right.$ area $\times 1 \mathrm{~m}$ slip for pure thrust motion against the relative plate motion; DeMets et al. 2010), which is composed of a triangular slip-rate function with a half-rise time of $0.25 \mathrm{~s}$ computed at a sampling frequency of $20 \mathrm{~Hz}$ (Fig. 1d). The model plane is a $190 \times 130 \mathrm{~km}^{2}$ rectangle with strike and dip angles of $2.7^{\circ}$ and $15^{\circ}$, respectively, that has been discretized for point sources with a spatial resolution of $2 \mathrm{~km} \times 2 \mathrm{~km}$ in both strike and dip directions. The initial rupture point (hypocentre) is located at $31.637^{\circ} \mathrm{S}, 71.741^{\circ} \mathrm{W}$, and $25-\mathrm{km}$ depth. Each point source is triggered to rupture by the expanding circular rupture front propagating at a constant speed of $3 \mathrm{~km} / \mathrm{s}$ from the hypocentre (Fig. 1c). The Green's function that is convolved with the slip-rate function to generate the synthetic waveforms is calculated by the method of Kikuchi \& Kanamori (1991). Near the source area, a layered medium (Table 1) is used for calculating the Haskell propagator matrix for the Green's function, and the ak135 model (Kennett et al. 1995) is used to calculate geometrical spreading factors, ray parameters, and travel times. The synthetic waveforms were calculated for the stations at teleseismic distances between $30^{\circ}-90^{\circ}$ (Fig. 1e), and the weighting factor 
Table 1. Near-source structure used to calculate Green's function.

\begin{tabular}{cccc}
$\begin{array}{c}V_{\mathrm{P}} \\
(\mathrm{km} / \mathrm{s})\end{array}$ & $\begin{array}{c}V_{\mathrm{S}} \\
(\mathrm{km} / \mathrm{s})\end{array}$ & $\begin{array}{c}\text { Density } \\
\left(10^{3} \mathrm{~kg} / \mathrm{cm}^{3}\right)\end{array}$ & $\begin{array}{c}\text { Thickness } \\
(\mathrm{km})\end{array}$ \\
\hline 1.50 & 0.00 & 1.02 & 4.00 \\
4.80 & 2.77 & 2.72 & 4.00 \\
5.50 & 3.18 & 2.72 & 4.00 \\
6.00 & 3.46 & 2.86 & 4.00 \\
6.40 & 3.70 & 2.86 & 6.00 \\
6.80 & 3.93 & 3.03 & 8.00 \\
7.80 & 4.32 & 3.42 & 0.00
\end{tabular}

$w_{j}$ in eqs. (1) and (7) is calculated according to the following rules:

$$
\begin{aligned}
w_{j} & =\frac{r_{j}}{\sum_{j} r_{j}}, \\
r_{j} & =\frac{1}{\sum_{j^{\prime}} m_{j j^{\prime}}}, \\
m_{j j^{\prime}} & = \begin{cases}1 & \left(\Delta_{j j^{\prime}} \leq 20^{\circ}\right) \\
0 & \left(\Delta_{j j^{\prime}}>20^{\circ}\right),\end{cases}
\end{aligned}
$$

where $\Delta_{j j^{\prime}}$ is the epicentral distance between a pair of stations. In the BP method, this weighting factor is based on globally distributed stations (e.g. Fan \& Shearer 2015), whereas when the BP method is applied to an array of stations, the weighting factor is represented by, for example, a cosine function (Ishii et al. 2007), and the weight given to the waveform decays with distance from the centre of the station array. Note that in this test, the hypocentre, fault geometry, station configuration, and nearsource velocity structures are those used in seismic source study of the $M_{\mathrm{W}} 8.32015$ Illapel, Chile, earthquake (Okuwaki et al. 2016). We used the BP and HBP methods to backproject the synthetic waveforms onto the fault. To show systematic bias independent of specific point-source distributions, we computed backprojection images for 100 cases of 20 randomly selected point sources (Fig. 1b).

We then plotted the backprojected signal intensities at the locations of point sources shown in Fig. 1(b) both in map view (Figs. 2a and b) and along depth (Figs. 3a and b). The result shows a clear depth dependence of the backprojected signal intensity on the model fault plane, where the signal intensity increases with the depth of the point sources even though we generated the synthetic waveforms by assuming the same potency at all the point sources. The mean values of the signal intensities measured within 5-km-depth bins monotonically increase from the shallowest to deepest bins; $0.52 \pm 0.13$ to $0.85 \pm 0.12$ and $0.55 \pm 0.10$ to $0.90 \pm 0.10$ for the original BP and HBP methods, respectively (Figs. $3 \mathrm{a}$ and $b$ ). This depth dependence in both the BP and HBP images reflects the depth dependence of the 
(a) BP (b) HBP (c) Kinematic BP

(d) Kinematic HBP
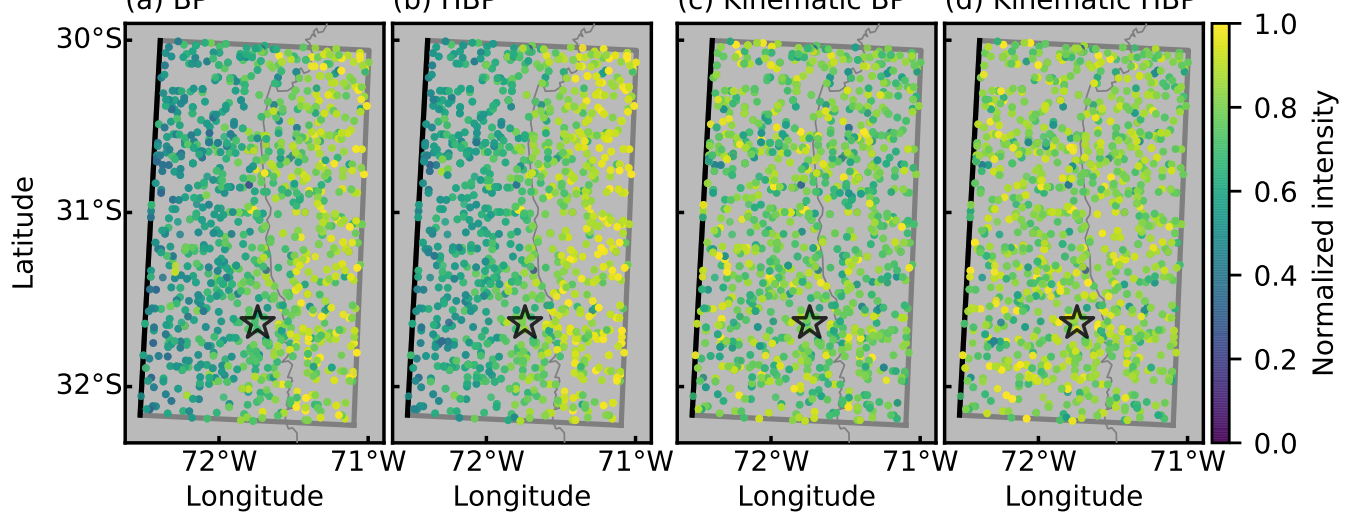

Figure 2. Map view of the result with the (a) original BP, (b) original HBP, (c) kinematic BP, and (d) kinematic HBP methods. Color represents the maximum intensity of the backprojected signal among the source duration, which is normalized by the maximum intensity among backprojected images for each case of \#1-\#100. The gray rectangle outlines the model fault plane, the black line is the up-dip limit of the fault area, and the star denotes the nucleation point (hypocentre). The thin gray curved line shows the coastline.

(a) BP

(c) Kinematic BP

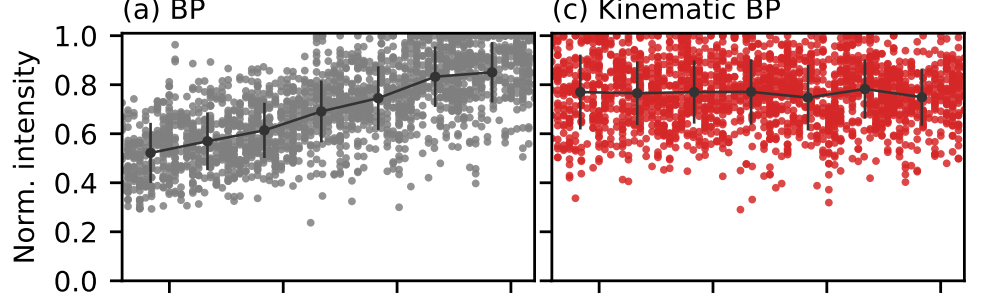

$\begin{array}{ll}\text { (b) HBP } & \text { (d) Kinematic HBP }\end{array}$

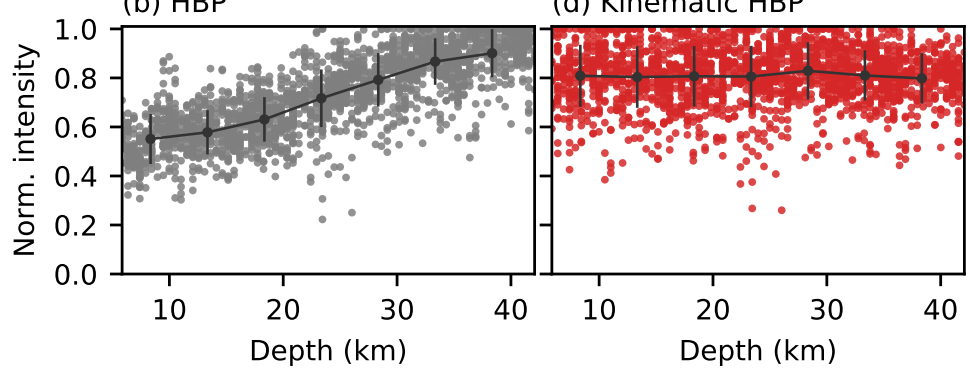

Figure 3. Depth view of the normalized intensity of images obtained by the (a) original BP, (b) original HBP, (c) kinematic BP, and (d) kinematic HBP methods. Abscissa is the depth of point sources, and the ordinate is the normalized intensity of the backprojected images. Black dot is the mean value of the signal intensities within each 5-km-depth bin. One standard deviation of the signal intensities within each depth bin is shown as the vertical error bar. 
fractions in eqs. (6) and (11), which have the Green's functions as their numerator and the normalizing factor as their denominator. In the assumed dipping model fault plane embedded in a layered medium (Table 1), the Green's function displays a depth dependence related to the rigidity of the medium and to geometrical spreading factors, both of which become larger with depth. Thus, we can conclude that the BP and HBP methods are inherently designed to retrieve contributions of wave radiation to entire waveforms, which should be proportional to the amplitude of the Green's functions. Note that the weighting factor $w_{j}$ fluctuates at most $9 \%$ for the station configuration adopted in this numerical test (Fig. 1e), and it does not significantly affect the signal intensity. The numerical test presented in this study extends the result of Fukahata et al. (2014) that did not rigorously consider the effect of a scaling factor due to the amplitude of the Green's function, because they basically considered the case of a single point source. However, for the case of multiple-point sources (Fig. 1), the dependence of the signal intensity on the source location has been clearly revealed (Figs. $2 a$ and $b$ and $3 a$ and $b$ ), because the scaling factor is proportional to the amplitude of the Green's function which depends on the source location shown in eqs. (6) and (11).

\section{NEW SCALING FACTORS FOR BP AND HBP METHODS TO IMAGE SLIP MOTION}

The depth dependence of the BP and HBP images (Figs. 2a and $b$ and 3a and $b$ ) highlights the inherent design of the BP and HBP methods, which extracts the relative strength of the wave radiation scaled by the amplitude of the Green's function; as a result, the BP and HBP images are biased when they are interpreted as the slip motion. However, BP and HBP methods are potentially useful for investigating the details of rupture properties or slip motion recorded in high-frequency waveforms. Therefore, it would be convenient if we can design BP and HBP methods to retrieve the slip motion on a fault plane from the backprojected images. For this purpose, we propose variants of the BP and HBP methods in which the normalizing factors in eqs. (1) and (7) of the original BP and HBP methods are appropriately modified. We then test the proposed methods in the same situation as described in Section 2.3 and also apply them to real waveforms of the $M_{\mathrm{W}} 8.32015$ Illapel, Chile, earthquake.

\subsection{Modification in kinematic BP and HBP methods and synthetic test}

In order to retrieve the slip motion from the signal intensity, we modify the BP and HBP methods by changing the normalizing factors to cancel out the numerators of the signal terms, $G_{i j}$ in eq. (6) and $G_{i j} \hat{\times} G_{i j}^{p}$ in eq. (11). For the BP method, we propose a normalizing factor $A_{i j}^{\mathrm{kBP}}$ as

$$
A_{i j}^{\mathrm{kBP}}=g_{i j}^{p}
$$



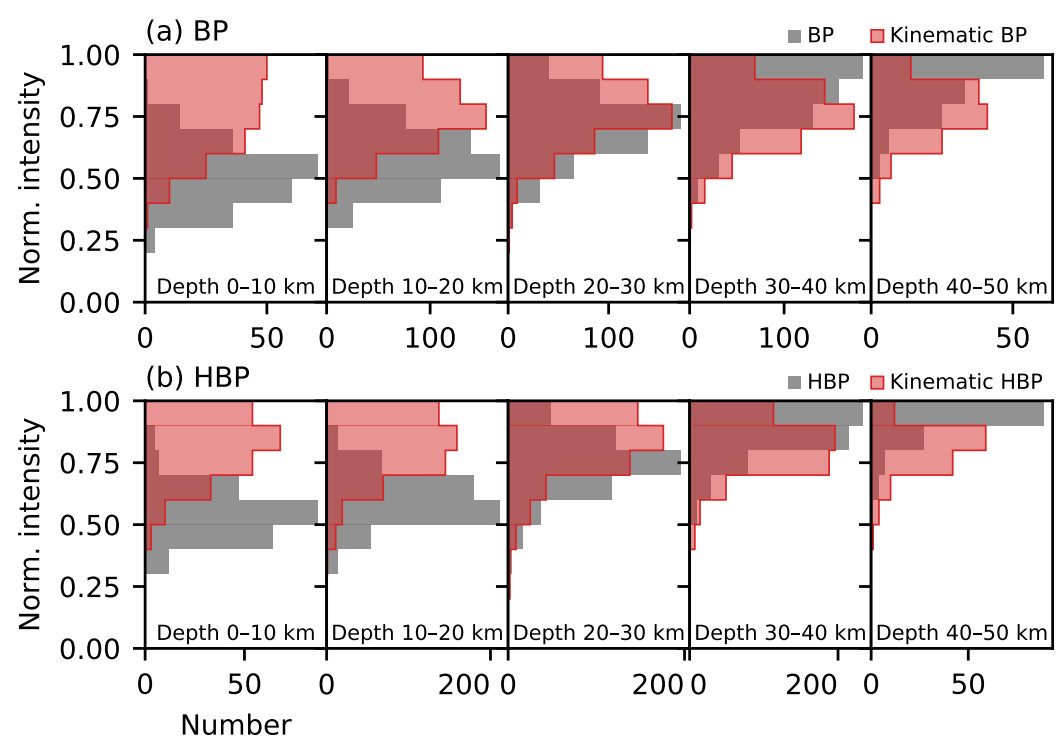

Figure 4. Histograms of the normalized intensity of the signals obtained by (a) the original and kinematic BP methods, and (b) the original and kinematic HBP methods.

where $g_{i j}^{p}$ is the amplitude of the first motion of the theoretical Green's function, which corresponds to the first local maximum (or minimum if polarity is down) of the amplitude from the arrival time. For the HBP method, we modify the normalizing factor $A_{i j}$ in eq. (8) by using the theoretical Green's function $G_{i j}^{p}$ :

$$
A_{i j}^{\mathrm{kHBP}}=\int_{0}^{T} G_{i j}^{p 2}\left(\tau+t_{i j}^{p}\right) d \tau
$$

The bias in the original BP and HBP images (Section 2.3) is expected to be corrected by this modification of the normalizing factors. Thus, the kinematic BP and HBP images should be more directly related to the slip motion on a fault.

The same numerical test that we carried out in Section 2.3 is performed to examine how the proposed normalization factors modify the resultant signal intensity. As shown in Figs. 2-4, the depth dependence seen in the original BP and HBP images has mostly disappeared; basically uniform distribution of signal intensity with depth is obtained by the kinematic BP and HBP methods. The mean values of the signal intensities within the shallowest bins have been increased by 1.48 and 1.47 with the kinematic BP and HBP methods, respectively (Fig. 3), compared to the mean values of the original results. These results indicate that the input potency used for generating the synthetic waveforms is backprojected onto the point source without the depth-dependent bias. 


\subsection{Theoretical background of kinematic BP and HBP methods}

Here, we consider the theoretical background of the kinematic BP and HBP methods, which successfully suppressed the depth-dependent bias, following the procedure taken in Fukahata et al. (2014) with the modified normalizing factors of eqs. (13) and (14).

In the kinematic BP method as well as in the original BP method, we first assume that the Green's function in eq. (6) is an impulsive response function (Fukahata et al. 2014), which can be represented by Dirac's delta function with the amplitude of the first motion of the Green's function as a scale factor on the basis that later phases and inelastic attenuation can be neglected:

$$
G_{i j}\left(t+t_{i j}\right) \approx g_{i j} \delta(t)
$$

where $g_{i j}$ is the amplitude of the first motion of the true Green's function, and $t_{i j}$ is the true travel time of the $P$ phase. If we can further assume that the true travel time $t_{i j}$ is approximately equal to the theoretical travel time of $t_{i j}^{p}$,

$$
t_{i j}^{p} \approx t_{i j}
$$

eq. (15) becomes

$$
G_{i j}\left(t+t_{i j}^{p}\right) \approx g_{i j} \delta(t)
$$

Substituting Eq. (17) into eq. (6), we obtain

$$
s_{i}^{\mathrm{kBP}}=\sum_{j} \frac{w_{j}}{A_{j}} a_{i} g_{i j}(t)
$$

If we can compute a Green's function for the $P$ phase that satisfies

$$
g_{i j}^{p} \approx g_{i j}
$$

the fraction of the Green's function and the normalizing factor in eq. (18) is canceled out in the kinematic BP method because we now have defined the normalizing factor as in eq. (13). Hence, the modified signal intensity at the $i$ th source location $s_{i}^{\mathrm{kBP}}$ becomes

$$
s_{i}^{\mathrm{kBP}}(t) \approx a_{i}(t)
$$

where we used $\sum_{j} w_{j}=1$. In eq. (20) the signal intensity in the kinematic BP method, $s_{i}^{\mathrm{kBP}}$, directly corresponds to the slip velocity $a_{i}$. Which of the Green's function due to unit-step slip velocity or displacement can well satisfy the assumption of eq. (17) is hard to be determined (Fukahata et al. 2014), but we note if the effect of the attenuation for the teleseismic body waves can be neglected, the slip motion $a_{i}$ corresponds to the slip acceleration for the velocity data $u_{j}$.

In the kinematic HBP method as well as in the original HBP method, we first assume that the 
theoretical Green's function is a good approximation of the true Green's function as

$$
G_{i j}^{p}(t) \approx G_{i j}(t)
$$

Substituting eq. (21) into eq. (11), we obtain

$$
s_{i}^{\mathrm{kHBP}}(t) \approx \sum_{j} w_{j} \frac{\left(\left(a_{i} * G_{i j}^{p}\right) \hat{\times} G_{i j}^{p}\right)(t)}{A_{i j}} .
$$

If we use the normalizing factor defined by eq. (14), the fraction that is composed of the crosscorrelation of the Green's functions and the normalizing factor in eq. (22) results in the normalized auto-correlation function of the Green's function. Therefore, if we can assume that the stacked autocorrelation functions is like the Dirac's delta function with certain amplitude (Fukahata et al. 2014), eq. (22) becomes

$$
\sum_{j} w_{j} \frac{\left(G_{i j}^{p} \hat{\times} G_{i j}^{p}\right)(t)}{\int_{0}^{T} G_{i j}^{p 2}\left(\tau+t_{i j}^{p}\right) d \tau} \approx \delta(t),
$$

where we used $\sum_{j} w_{j}=1$. Then, the modified signal intensity at the $i$ th source location $s_{i}^{\mathrm{kHBP}}$ is finally expressed as

$$
s_{i}^{\mathrm{kHBP}}(t) \approx a_{i}(t)
$$

In eq. (24) the signal intensity of the kinematic HBP image, $s_{i}^{\mathrm{kHBP}}$, directly corresponds to the slip velocity $a_{i}$.

\subsection{Application to the real data}

In order to demonstrate how the relative signal intensity is modified by the kinematic BP and HBP methods for the real case, we applied them to the waveforms of the $M_{\mathrm{W}} 8.32015$ Illapel, Chile, earthquake with the same procedure adopted by Okuwaki et al. (2016), where they used the original HBP method (Fig. 5). Here, 4th root stacking (e.g. McFadden et al. 1986) was used to enhance the signalto-noise ratio of the image, and the data were filtered in the $0.3-2.0 \mathrm{~Hz}$ frequency band. Compared with the original BP and HBP methods, the signal intensities of the kinematic BP and HBP methods in the region shallower than $25-\mathrm{km}$ depth have been increased by 1.25 and 1.33 of their mean values, respectively (Figs. $5 \mathrm{c}$ and f), which are similar to the ones for the numerical tests measured within 0 to $25-\mathrm{km}$-depth bin; 1.28 and 1.31 . We note that the mean values of the real applications and the numerical tests may not be simply compared since for the real applications, the mean values were measured among all the point sources in the model fault, while for the numerical tests, we only used the intensities on the input-source points (Fig. 1b). Based on the numerical test, we can consider that the depth-dependent bias included in the original BP and HBP method is successfully suppressed by 

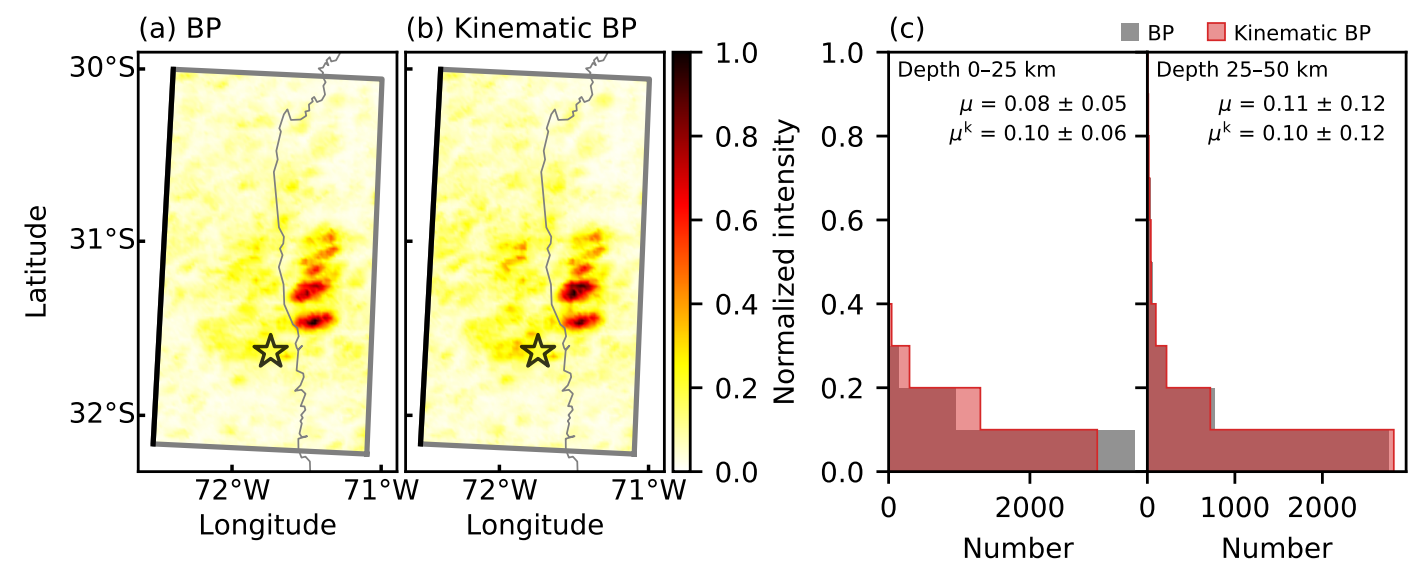

(d) HBP

(e) Kinematic HBP
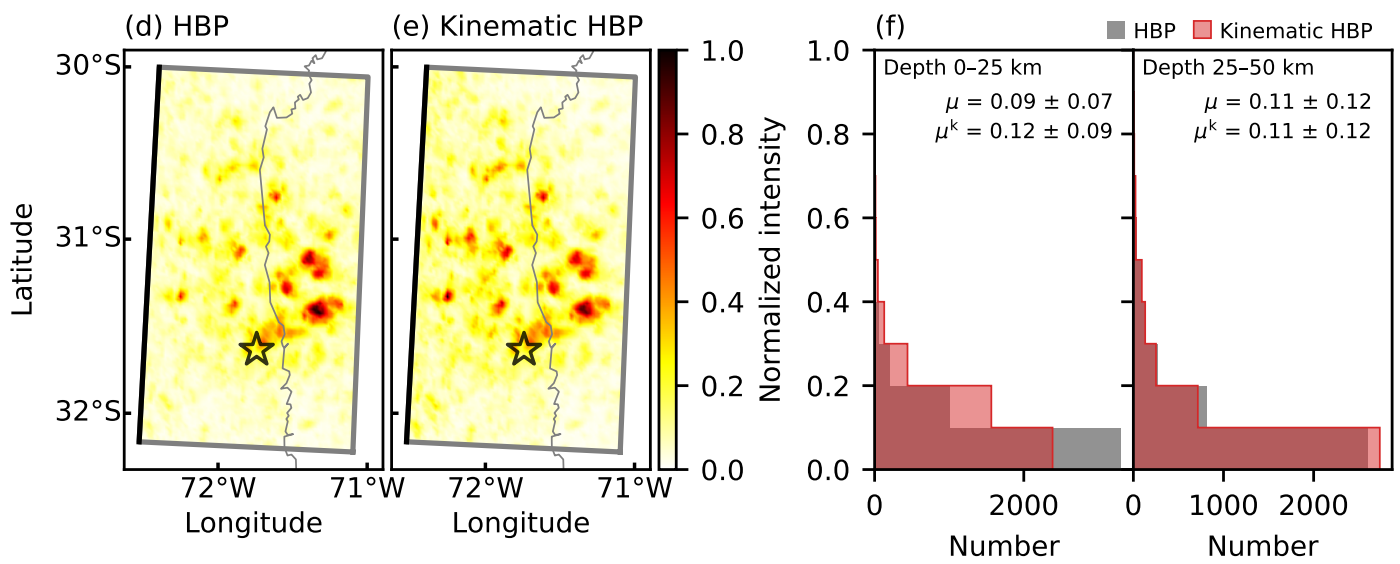

Figure 5. Comparison of the real-data application of the original and the kinematic BP and HBP methods for the $M_{\mathrm{W}} 8.32015$ Illapel Chile earthquake. (a, b, d, e) Color represents the normalized intensity of the backprojected signal. The gray rectangle outlines the model fault plane with a dip angle of $15^{\circ}$, the black line on the west side is the up-dip limit of the fault area, and the star denotes the nucleation point (hypocentre). The thin gray curve shows the coastline. (c, f) Histograms of the normalized intensity of the signals obtained by the original and the kinematic BP and HBP methods. $\mu$ and $\mu^{\mathrm{k}}$ are the mean values of the original and the kinematic BP and HBP signal intensities, respectively.

the kinematic BP and HBP methods. The source models of the 2015 Illapel earthquake showed a complex rupture evolution along both the up-dip and down-dip directions (Melgar et al. 2016; An et al. 2017; Yin et al. 2018; Meng et al. 2018), which is more consistent with the image obtained by the kinematic BP and HBP methods. The increased signal in the shallow part should reflect the source of high-frequency radiation from the up-dip rupture propagation. The general difference between the BP and HBP images, especially in the shallow region, reflects the enhancement of the spatial resolution in dip direction of the HBP method by adopting the information of depth phases of the Green's function (Yagi et al. 2012). While in the region deeper than 25-km depth, the distributions of the signal intensi- 
ties remain almost the same for the original and kinematic BP and HBP methods (Figs. $5 \mathrm{c}$ and f). This suggests that the previous discussion of the deep-intense high-frequency radiation, which may result from the rupture of small high-stress patches that accelerate the following large slip (e.g. Okuwaki et al. 2016), should not be affected significantly even by the kinematic BP and HBP methods, at least in the case of the 2015 Illapel earthquake. The real applications suggest that the heterogeneity of the fracture energy or stress drop that perturbs the rupture-front propagation (e.g. Husseini et al. 1975; Fukuyama \& Madariaga 1998) may exist in both shallow and deep part of the fault, though the degree of heterogeneity may be different depending on depth.

\section{DISCUSSION}

In this study, we proposed the variants of the BP and HBP methods in order to directly retrieve the slip motion from the backprojected images by modifying the normalizing factors adopted in the original BP and HBP methods, which suppresses the depth-dependent bias in the original BP and HBP methods. However, for subduction zone megathrust earthquakes, the depth dependence of the signal intensity in the original BP and HBP images is still crucial information for assessing future-earthquake damage due to strong shaking, because buildings have resonant frequencies that lie in the frequency band used for the BP analyses (e.g. Snieder \& Şafak 2006; Meng et al. 2012a). The contribution from highfrequency wave radiation by a unit slip in the deeper part of the seismogenic zone tends to be larger than that from the radiation in the shallower part of the fault, provided that the fault is dipping and the rigidity of the medium increases with depth. Both the mathematical representations and numerical test indicate that in the original BP and HBP methods the signal intensity does not directly correspond to the slip behaviour.

We verified through numerical tests that the kinematic BP and HBP methods worked well for suppressing depth dependence of the signal intensity and extracting slip motion on a fault surface, which should be useful for unbiased interpretation of high-frequency signals in the context of the source-rupture evolution. However, even if we use the kinematic BP and HBP methods, in the ideal model setting to generate the synthetic waveforms, the input sources were still imperfectly recovered, and the normalized backprojection intensity ranged from about 0.5 to 1.0 (Figs. 3 and 4). One possible reason for this spread in the distribution is due to the noise given in the second terms in eqs. (4) and (9). We assumed for both the original and kinamatic BP and HBP methods that the noise terms can be suppressed by stacking as eqs. (5) and (10) based on Fukahata et al. (2014). However, in our numerical test, where the multiple-point sources with a uniform slip-rate function are randomly distributed (Fig. 1), the interference of the noise and signal terms cannot be neglected when the sources are close to each other since the convolution functions of the slip velocity and the Green's function get similar 
among the neighbouring locations. As a result, the signal intensity may be intensified or decreased by the noise, and the signal intensity at the specific source point may not exactly represent the slip. This imperfect recovery of the input sources is a common feature in both the original and kinematic BP and HBP methods, irrespective of the implementation of the normalizing factor because this comes from the resemblance of the Green's function at sources (Fukahata et al. 2014). We also note that the assumptions we made in eqs. (19) and (21) may not work efficiently for the analysis of real data, because of the limitation to calculate the Green's function accurately (e.g. Yagi \& Fukahata 2011), and the uncertainty of the depth and focal mechanism of the source points may distort the kinematic BP and HBP images for real applications.

We have discussed the depth dependence of the signal intensity in the original BP and HBP methods by only considering that the scaling factors include the Green's functions in the numerators of the signal terms in eqs. (4) and (9). However the normalizing factors adopted in the original BP and HBP methods also include the slip motion $a_{i}$ since the normalizing factors are composed of the waveform data $u_{j}$ (eq. 3). For example, eq. (6) can be rewritten as

$$
s_{i}^{\mathrm{BP}}(t) \approx \sum_{j} w_{j} \frac{\left(a_{i} * G_{i j}\right)\left(t+t_{i j}^{p}\right)}{\operatorname{pol}\left(u_{j}\right) \cdot \sqrt{\int_{0}^{T}\left[\sum_{i^{\prime}}\left(a_{i^{\prime}} * G_{i^{\prime} j}\right)\left(\tau+t_{j}^{p}\right)\right]^{2} d \tau}},
$$

for the original BP method, in which $a_{i}$ is included in both the numerator and denominator. Thus, the relationship between the resultant signal intensity and the slip motion $a_{i}$ is essentially non-linear in the original BP and HBP method, which makes it difficult to understand the effect of the Green's function on the depth dependence of the signal intensity. On the other hand, the normalizing factors in the kinematic BP and HBP methods are designed to include the Green's function alone. Because of that, the signal intensity is directly related to the slip motion, which establishes a linear relation between the signal intensity and the slip motion.

\section{CONCLUSION}

Through the mathematical reviews and numerical tests of the time-domain BP and HBP methods, we confirmed that the signal intensity in the original BP and HBP methods was designed to retrieve the relative strength of wave radiation. Therefore, the signal intensity is proportional to the amplitude of the Green's function, which leads to clear depth dependence of the signal intensity. The depth dependence of the BP and HBP images gives a bias when the signal intensity of the image is interpreted as the rupture property. On the other hand, the kinematic BP and HBP methods, the variants of the BP techniques proposed in this paper, can directly relate the signal intensity to slip motion on a fault surface, which is free from the depth dependence. The proposed methods are useful for quantifying 
the depth dependence of the high-frequency sources, which would deepen our understanding of the high-frequency radiation and its role on the rupture property during an earthquake.

\section{ACKNOWLEDGMENTS}

We appreciate the editor Martin Mai and the anonymous reviewer and Zengxi Ge for their thorough review and constructive comments and suggestions that significantly improved our paper. This work was supported by Grant-in-aid for Japan Society for the Promotion of Science (JSPS) Research Fellow (JP16J00298) and JSPS Grants-in-aid for Scientific Research (JP16K05529, JP16H01842, and JP16K05539). The authors thank Hisahiko Kubo, Lingsen Meng, Takahiko Uchide, and Wenyuan Fan for their valuable comments on the early stage of this study. Teleseismic waveforms from the networks; Antarctic Seismographic Argentinean Italian Network (AI; https://doi.org/10.7914/SN/AI), Canadian National Seismograph Network (CN; https://doi.org/10.7914/SN/CN), GEOSCOPE (G; https://doi.org/10.18715/GEOSC Global Telemetered Seismograph Network (USAF/USGS, GT; https://doi.org/10.7914/SN/GT), Global Seismograph Network (GSN IRIS/IDA, II; https://doi.org/10.7914/SN/II), and Global Seismograph Network (GSN IRIS/USGS, IU; https://doi.org/10.7914/SN/IU) are downloaded through the IRISDMC (https://ds.iris.edu/ds/nodes/dmc/). The figures are generated with matplotlib (Hunter 2007, Version v2.2.2; https://doi.org/10.5281/zenodo.1202077). All the data and results shown in this study are stored in Github (https://github.com/rokuwaki).

\section{REFERENCES}

Aki, K., 1979. Characterization of barriers on an earthquake fault, J. Geophys. Res., 84(B11), 6140.

An, C., Yue, H., Sun, J., Meng, L., \& Báez, J. C., 2017. The 2015 Mw 8.3 Illapel, Chile, Earthquake: DirectionReversed Along-Dip Rupture with Localized Water Reverberation, Bull. Seismol. Soc. Am., 107(5), 24162426.

Beresnev, I. A., 2017. Factors controlling high-frequency radiation from extended ruptures, J. Seismol., 21(5), $1277-1284$.

Bernard, P. \& Madariaga, R., 1984. A new asymptotic method for the modeling of near-field accelerograms, Bull. Seismol. Soc. Am., 74(2), 539-557.

Bruhat, L., Fang, Z., \& Dunham, E. M., 2016. Rupture complexity and the supershear transition on rough faults, J. Geophys. Res. Solid Earth, 121(1), 210-224.

D’Amico, S., Koper, K. D., Herrmann, R. B., Akinci, A., \& Malagnini, L., 2010. Imaging the rupture of the Mw 6.3 April 6, 2009 L'Aquila, Italy earthquake using back-projection of teleseismic P-waves, Geophys. Res. Lett., 37(3), 1-5.

Das, S. \& Aki, K., 1977. Fault plane with barriers: A versatile earthquake model, J. Geophys. Res., 82(36), $5658-5670$. 
DeMets, C., Gordon, R. G., \& Argus, D. F., 2010. Geologically current plate motions, Geophys. J. Int., 181(1), $1-80$.

Dunham, E. M., Belanger, D., Cong, L., \& Kozdon, J. E., 2011. Earthquake Ruptures with Strongly RateWeakening Friction and Off-Fault Plasticity, Part 2: Nonplanar Faults, Bull. Seismol. Soc. Am., 101(5), 23082322.

Fan, W. \& Shearer, P. M., 2015. Detailed rupture imaging of the 25 April 2015 Nepal earthquake using teleseismic P waves, Geophys. Res. Lett., 42(14), 5744-5752.

Fan, W. \& Shearer, P. M., 2016. Local near instantaneously dynamically triggered aftershocks of large earthquakes, Science, 353(6304), 1133-1136.

Fan, W. \& Shearer, P. M., 2017. Investigation of Backprojection Uncertainties With M6 Earthquakes, J. Geophys. Res. Solid Earth, 122(10), 7966-7986.

Fan, W., Shearer, P. M., Ji, C., \& Bassett, D., 2016. Multiple branching rupture of the 2009 Tonga-Samoa earthquake, J. Geophys. Res. Solid Earth, 121(8), 5809-5827.

Fan, W., Bassett, D., Jiang, J., Shearer, P. M., \& Ji, C., 2017. Rupture evolution of the 2006 Java tsunami earthquake and the possible role of splay faults, Tectonophysics, 721, 143-150.

Fukahata, Y., Yagi, Y., \& Rivera, L., 2014. Theoretical relationship between back-projection imaging and classical linear inverse solutions, Geophys. J. Int., 196(1), 552-559.

Fukuyama, E. \& Madariaga, R., 1998. Rupture dynamics of a planar fault in a 3d elastic medium: Rate- and slip-weakening friction, Bull. Seismol. Soc. Am., 88(1), 1.

Hunter, J. D., 2007. Matplotlib: A 2D Graphics Environment, Comput. Sci. Eng., 9(3), 90-95.

Husseini, M. I., Jovanovich, D. B., Randall, M. J., \& Freund, L. B., 1975. The fracture energy of earthquakes, Geophys. J. Int., 43(2), 367-385.

Ishii, M., Shearer, P. M., Houston, H., \& Vidale, J. E., 2005. Extent, duration and speed of the 2004 SumatraAndaman earthquake imaged by the Hi-Net array, Nature, 435(7044), 933-936.

Ishii, M., Shearer, P. M., Houston, H., \& Vidale, J. E., 2007. Teleseismic P wave imaging of the 26 December 2004 Sumatra-Andaman and 28 March 2005 Sumatra earthquake ruptures using the Hi-net array, J. Geophys. Res. Solid Earth, 112(11), 1-16.

Kennett, B. L. N., Engdahl, E. R., \& Buland, R., 1995. Constraints on seismic velocities in the Earth from traveltimes, Geophys. J. Int., 122(1), 108-124.

Kikuchi, M. \& Kanamori, H., 1991. Inversion of complex body waves-III, Bull. Seismol. Soc. Am., 81(6), 2335-2350.

Kiser, E. \& Ishii, M., 2011. The $2010 \mathrm{mw} 8.8$ chile earthquake: Triggering on multiple segments and frequency-dependent rupture behavior, Geophys. Res. Lett., 38(7).

Kiser, E. \& Ishii, M., 2013. Hidden aftershocks of the 2011 Mw 9.0 Tohoku, Japan earthquake imaged with the backprojection method, J. Geophys. Res. Solid Earth, 118(10), 5564-5576.

Kiser, E. \& Ishii, M., 2017. Back-Projection Imaging of Earthquakes, Annu. Rev. Earth Planet. Sci., 45(1), 271-299. 
Kiser, E., Ishii, M., Langmuir, C. H., Shearer, P. M., \& Hirose, H., 2011. Insights into the mechanism of intermediate-depth earthquakes from source properties as imaged by back projection of multiple seismic phases, J. Geophys. Res. Solid Earth, 116(6), 1-26.

Koper, K. D., Hutko, A. R., Lay, T., \& Sufri, O., 2012. Imaging short-period seismic radiation from the 27 February 2010 Chile (MW 8.8) earthquake by back-projection of P, PP, and PKIKP waves, J. Geophys. Res. Solid Earth, 117(B2), 1-16.

Lay, T., Kanamori, H., Ammon, C. J., Koper, K. D., Hutko, A. R., Ye, L., Yue, H., \& Rushing, T. M., 2012. Depth-varying rupture properties of subduction zone megathrust faults, J. Geophys. Res. Solid Earth, 117(B4), 1-12.

Madariaga, R., 1977. High-frequency radiation from crack (stress drop) models of earthquake faulting, Geophys. J. Int., 51(3), 625-651.

Madariaga, R., Ampuero, J. P., \& Adda-Bedia, M., 2006. Seismic radiation from simple models of earthquakes, in Earthquakes Radiated Energy Phys. Faulting, no. 1999, pp. 223-236.

Mai, P. M., Galis, M., Thingbaijam, K. K. S., Vyas, J. C., \& Dunham, E. M., 2017. Accounting for Fault Roughness in Pseudo-Dynamic Ground-Motion Simulations, Pure Appl. Geophys., 174(9), 3419-3450.

McFadden, P. L., Drummond, B. J., \& Kravis, S., 1986. The Nth-root stack: Theory, applications, and examples, Geophysics, 51(10), 1879-1892.

Melgar, D., Fan, W., Riquelme, S., Geng, J., Liang, C., Fuentes, M., Vargas, G., Allen, R. M., Shearer, P. M., \& Fielding, E. J., 2016. Slip segmentation and slow rupture to the trench during the 2015, M w 8.3 Illapel, Chile earthquake, Geophys. Res. Lett., 43(3), 961-966.

Meng, L., Inbal, A., \& Ampuero, J.-P., 2011. A window into the complexity of the dynamic rupture of the 2011 Mw 9 Tohoku-Oki earthquake, Geophys. Res. Lett., 38(7), 1-6.

Meng, L., Ampuero, J.-P., Sladen, A., \& Rendon, H., 2012a. High-resolution backprojection at regional distance: Application to the Haiti M 7.0 earthquake and comparisons with finite source studies, J. Geophys. Res. Solid Earth, 117(B4), 1-16.

Meng, L., Ampuero, J.-P., Stock, J., Duputel, Z., Luo, Y., \& Tsai, V. C., 2012b. Earthquake in a Maze: Compressional Rupture Branching During the 2012 Mw 8.6 Sumatra Earthquake, Science, 337(6095), 724726.

Meng, L., Zhang, A., \& Yagi, Y., 2016. Improving back projection imaging with a novel physics-based aftershock calibration approach: A case study of the 2015 Gorkha earthquake, Geophys. Res. Lett., 43(2), 628-636.

Meng, L., Bao, H., Huang, H., Zhang, A., Bloore, A., \& Liu, Z., 2018. Double pincer movement: Encircling rupture splitting during the 2015 mw 8.3 illapel earthquake, Earth Planet. Sci. Lett., 495, 164 - 173.

Okuwaki, R. \& Yagi, Y., 2018. Role of geometric barriers in irregular-rupture evolution during the 2008 Wenchuan earthquake, Geophys. J. Int., 212(3), 1657-1664.

Okuwaki, R., Yagi, Y., \& Hirano, S., 2014. Relationship between High-frequency Radiation and Asperity Ruptures, Revealed by Hybrid Back-projection with a Non-planar Fault Model, Sci. Rep., 4(1), 7120. 
Okuwaki, R., Yagi, Y., Aránguiz, R., González, J., \& González, G., 2016. Rupture Process During the 2015 Illapel, Chile Earthquake: Zigzag-Along-Dip Rupture Episodes, Pure Appl. Geophys., 173(4), 1011-1020.

Satriano, C., Kiraly, E., Bernard, P., \& Vilotte, J.-P., 2012. The 2012 Mw 8.6 Sumatra earthquake: Evidence of westward sequential seismic ruptures associated to the reactivation of a N-S ocean fabric, Geophys. Res. Lett., 39(15), 1-6.

Satriano, C., Dionicio, V., Miyake, H., Uchida, N., Vilotte, J. P., \& Bernard, P., 2014. Structural and thermal control of seismic activity and megathrust rupture dynamics in subduction zones: Lessons from the Mw 9.0, 2011 Tohoku earthquake, Earth Planet. Sci. Lett., 403, 287-298.

Shi, Z. \& Day, S. M., 2013. Rupture dynamics and ground motion from 3-D rough-fault simulations, $J$. Geophys. Res. Solid Earth, 118(3), 1122-1141.

Snieder, R. \& Şafak, E., 2006. Extracting the building response using seismic interferometry: Theory and application to the Millikan Library in Pasadena, California, Bull. Seismol. Soc. Am., 96(2), 586-598.

Spudich, P. \& Frazer, L., 1984. Use of ray theory to calculate high-frequency radiation from earthquake sources having spatially variable rupture velocity and stress drop, Bull. Seismol. Soc. Am., 74(6), 2061-2082.

Suzuki, M. \& Yagi, Y., 2011. Depth dependence of rupture velocity in deep earthquakes, Geophys. Res. Lett., 38(5), 1-5.

Uchide, T., Yao, H., \& Shearer, P. M., 2013. Spatio-temporal distribution of fault slip and high-frequency radiation of the 2010 El Mayor-Cucapah, Mexico earthquake, J. Geophys. Res. Solid Earth, 118(4), 15461555 .

Vallée, M. \& Satriano, C., 2014. Ten year recurrence time between two major earthquakes affecting the same fault segment, Geophys. Res. Lett., 41(7), 2312-2318.

Wang, D. \& Mori, J., 2011. Frequency-dependent energy radiation and fault coupling for the $2010 \mathrm{Mw} 8.8$ Maule, Chile, and 2011 Mw9.0 Tohoku, Japan, earthquakes, Geophys. Res. Lett., 38(22), 1-7.

Yagi, Y. \& Fukahata, Y., 2011. Introduction of uncertainty of Green's function into waveform inversion for seismic source processes, Geophys. J. Int., 186(2), 711-720.

Yagi, Y. \& Okuwaki, R., 2015. Integrated seismic source model of the 2015 Gorkha, Nepal, earthquake, Geophys. Res. Lett., 42(15), 6229-6235.

Yagi, Y., Nakao, A., \& Kasahara, A., 2012. Smooth and rapid slip near the Japan Trench during the 2011 Tohoku-oki earthquake revealed by a hybrid back-projection method, Earth Planet. Sci. Lett., 355-356, 94 101.

Yao, H., Shearer, P. M., \& Gerstoft, P., 2013. Compressive sensing of frequency-dependent seismic radiation from subduction zone megathrust ruptures, Proc. Natl. Acad. Sci., 110(12), 4512-4517.

Ye, L., Lay, T., Kanamori, H., \& Koper, K. D., 2013. Energy release of the 2013 Mw 8.3 Sea of Okhotsk earthquake and deep slab stress heterogeneity, Science, 341(6152), 1380-1384.

Ye, L., Lay, T., Kanamori, H., \& Rivera, L., 2016. Rupture characteristics of major and great ( Mw $\geq 7.0$ ) megathrust earthquakes from 1990 to 2015: 2. Depth dependence, J. Geophys. Res. Solid Earth, 121(2), 845-863. 
Yin, J., Yang, H., Yao, H., \& Weng, H., 2016. Coseismic radiation and stress drop during the 2015 Mw 8.3 Illapel, Chile megathrust earthquake, Geophys. Res. Lett., 43(4), 1520-1528.

Yin, J., Denolle, M. A., \& Yao, H., 2018. Spatial and Temporal Evolution of Earthquake Dynamics: Case Study of the Mw 8.3 Illapel Earthquake, Chile, J. Geophys. Res. Solid Earth, pp. 1-24. 